\title{
Epistemic games: a multi-layered task analysis
}

\author{
Michael Vignal, and Elizabeth Gire \\ Department of Physics, Oregon State University, Corvallis, OR 97331
}

\begin{abstract}
Physics education researchers have successfully employed the epistemic games framework as a descriptive framework for episodes of physics problem solving. Researchers have used this framework to analyze behaviors and activities at a variety of grain sizes. We conduct an analysis of layers of epistemic games of different grain size in student reasoning and behavior during a group interview of upper-division physics students plotting a 2D vector field. In our analysis, we propose three new epistemic games: Pick, Plug, and Plot; Plotting; and Answer Making with Sensemaking. We discuss how analyzing the interview with each of these games provides insight into the students' reasoning and how considering these games together in a multi-layered analysis yields additional insights.
\end{abstract}

\section{INTRODUCTION}

Collins and Ferguson [1] introduce the epistemic games framework as a tool for understanding the ways people "make sense of phenomena of the world." Tuminaro and Redish [2] adapt this framework to describe how introductory physics students solve physics problems. Researchers have since employed epistemic games at various grain sizes to a variety of physics contexts [3-7]. We employ the epistemic games framework to analyze an interview in which three upper-division undergraduate physics students plot a two-dimensional vector field. In this analysis, we consider 1) if the students engage in any yet unidentified epistemic games, and 2) if we can better understand the students' actions and behaviors by expanding on the idea of grain size to including multiple, concurrent epistemic games in our analysis.

In section IV, we describe three new epistemic games: Pick, Plug, and Plot (PPP); Plotting; and Answer Making with Sensemaking. In section $\mathrm{V}$, we simultaneously consider these three layers of games to better analyze and understand the students actions and behaviors.

\section{THE EPISTEMIC GAMES FRAMEWORK}

Collins and Ferguson [1] describe epistemic games as a "set of strategies and rules that guide inquiry." Epistemic games consist of entry and ending conditions, forms, and moves $[1,2]$ : entry and ending conditions trigger the start and end of an epistemic game; the epistemic form is the guiding structure that aids in the completion of the task [1]; and moves are the activation of resources or 'chunks' of commonly associated knowledge structures [2, 8].

Researchers employ the epistemic games framework at different layers of analysis. Tuminaro and Redish describe games (eg. Mapping Meaning to Mathematics and Recursive Plug and Chug) that can span a whole problem solving episode [2]. Kustusch et al. identify several epistemic games in advanced thermodynamics (eg. Partial Derivatives) that only describe part of a problem solving activity but may be specific instances or "sub-games" of Mapping Meaning to Mathematics or other games identified by Tuminaro and Redish [5]. Chen et al. identify the Answer Making Epistemic Game, which they believe could contain some of the games identified by Tuminaro and Redish as sub-games [4, 7].

Some researchers use the term grain size to describe these different layers $[3,6]$. We reserve the term grain size for describing different 'resolutions' of games that are ontologically similar, and we use the term layers to more generally describe such simultaneously occurring games. For example, we consider discussing Mapping Meaning to Mathematics and Answer Making Epistemic Game in terms of grain size is inappropriate, since the games have different goals. However, we still find it useful to compare and contrast these different layers of games.

\section{METHODS}

Our data comes from one group interview with three upper-division undergraduate physics majors (referred to in this report by pseudonyms) at a four-year public research-intensive university. The interviewer asked the group to "plot the vector field $\ldots f(x, y)=y \hat{\mathrm{i}}+x \hat{\mathrm{j}}$."

The interview was video recorded and transcribed. We categorized patterns in the students' actions with an open coding process that emerged naturally from the data [9], and we resolved these codes into epistemic games and moves. We did not seek inter-rater reliability in our coding, rather the codes were agreed upon by consensus.

This interview was originally conducted for the purpose of curricular development, so we, the researchers, had no part in constructing the interview tasks. This was an extra curricular activity for these students, who had worked with vector fields in previous electricity and magnetism courses.

\section{INDIVIDUAL EPISTEMIC GAME LAYERS}

We identify three new epistemic games: the smallgrained Pick, Plug, Plot (PPP); the medium-grained Plotting; and Answer Making with Sensemaking (AMS), a sensemaking elaboration of the Answer Making Epistemic Game identified by Chen et al. [4]. Table I includes our transcript spanning four minutes of discussion during 
TABLE I. Transcript and identified epistemic moves from the Pick Plug Plot (PPP), Plotting, and AMS epistemic games. This transcript spans four minutes of discussion.

\begin{tabular}{|c|c|c|c|c|c|}
\hline \# & Speaker & Transcript & PPP & Plotting & AMS \\
\hline 6 & Int.: & I want you to plot the vector field $f(x, y)=y \hat{\mathrm{i}}+x \hat{\mathrm{j}}$. & & Entry Cond. & Entry Cond. \\
\hline 7 & Ben: & OK. So one big axis then [draws a set of axes]. & & Identify & \multirow{4}{*}{$\begin{array}{l}\text { Conceptual } \\
\text { Reasoning }\end{array}$} \\
\hline 8 & Ian: & Yeah. & & Form & \\
\hline $\begin{array}{r}9 \\
10\end{array}$ & $\begin{array}{l}\text { Ben: } \\
\text { Ian: }\end{array}$ & $\begin{array}{l}\text { So, I think we should just plot a couple points to get the feel of it. } \\
\text { Yeah. }\end{array}$ & Entry Cond. & & \\
\hline 11 & Ben: & So let's plot $(0,1)$ [draws dot at $(0,1)]$ & Pick & \multirow{3}{*}{ PPP } & \\
\hline 12 & Ben: & So this means that we're going to have zero for i-hat and one for $\mathrm{j}$-hat. & Plug & & \multirow{2}{*}{ Do Math } \\
\hline 13 & Ben: & So it's going to go up like this [draws vector pointing in the positive y direction]. & Plot & & \\
\hline 14 & Ben: & $\begin{array}{l}\text { And we know the other point, at }(-1,0) \text {, should go this way [without looking at } \\
\text { formula, draws vector pointing in the negative y direction]. }\end{array}$ & & Symmetry & Sensemaking \\
\hline 48 & Ian: & And then let's try $\ldots(1,-1)$. & Pick & \multirow{10}{*}{\begin{tabular}{|l|l|} 
Symmetry \\
PPP
\end{tabular}} & $\begin{array}{l}\text { Conceptual } \\
\text { Reasoning }\end{array}$ \\
\hline 49 & Ben: & $\begin{array}{l}(1,-1) ? \text { So then we get [pause while they all look at formula] one in the j-hat and } \\
\text { negative one in the i-hat direction. }\end{array}$ & Plug & & \multirow{9}{*}{ Do Math } \\
\hline $\begin{array}{l}50 \\
51\end{array}$ & $\begin{array}{l}\text { Ian: } \\
\text { Ben: }\end{array}$ & $\begin{array}{l}\text { [Points with finger.] So it just points back in. } \\
\text { Points in. [Draws vectors from }(1,-1) \text { and }(-1,1) \text { towards origin.] }\end{array}$ & Plot & & \\
\hline 52 & Matt: & [Nodding.] Yeah, yeah. & & & \\
\hline 53 & Ian: & OK. Yeah. & & & \\
\hline 54 & Ben: & And those are length one. & \multirow{5}{*}{ Plot } & & \\
\hline 55 & Ian: & Yeah. & & & \\
\hline 56 & Ben: & Not square root of two. [Inaudible]. & & & \\
\hline 57 & Ian: & $\mathrm{Bu}-\mathrm{OK}$ & & & \\
\hline 58 & Matt: & [Nodding.] & & & \\
\hline 59 & Ben: & So what we see is, whenever we lie on this axis... & \multirow[b]{6}{*}{ Plot } & Extrapolate & Sensemaking \\
\hline 60 & Int.: & Par- uh, what length are they? & & & \\
\hline 61 & Ben: & What was that? & & & \\
\hline 62 & Int.: & What length are they? & & & \\
\hline $\begin{array}{l}63 \\
64\end{array}$ & $\begin{array}{l}\text { Ben: } \\
\text { Ian: }\end{array}$ & $\begin{array}{l}\text { [Looking at the plot.] Ah, you're right. They are square root of two. Because ... } \\
\text { These two [points at vectors Ben just drew]. }\end{array}$ & & \multirow{2}{*}{$\begin{array}{l}\text { PPP } \\
\text { and } \\
\text { Symmetry }\end{array}$} & \multirow[b]{2}{*}{ Do Math } \\
\hline 65 & Ben: & $\begin{array}{l}\text { Yeah, square root of two. So, actually, yeah, they should point all the way back. } \\
\text { It should touch the origin [redraws vectors extending to the origin]. } \\
\text { So they should ... yeah. }\end{array}$ & & & \\
\hline 67 & Ben: & $\begin{array}{l}\text { OK, yeah. And then so ... these vectors [on the positive } \mathrm{x} \text { and } \mathrm{y} \text { axes] will start } \\
\text { like pointing this way [up and to the right, parallel to the line } \mathrm{y}=\mathrm{x} \text {.] }\end{array}$ & & \multirow{5}{*}{ Extrapolate } & \multirow{5}{*}{ Sensemaking } \\
\hline 68 & Ian: & [Nodding.] & & & \\
\hline 69 & Ben: & These [on the negative $\mathrm{x}$ and $\mathrm{y}$ axes] will go that way [down and to the left.] & & & \\
\hline 70 & Ben: & $\begin{array}{l}\text { And these [in quadrant II] will go in [towards the origin] and this [in quadrant } \\
\text { IV] will ... those will go in as well. }\end{array}$ & & & \\
\hline 71 & Ian: & [Mm-hmm.] & & & \\
\hline $\begin{array}{l}72 \\
73\end{array}$ & $\begin{array}{l}\text { Ben: } \\
\text { Ian: }\end{array}$ & $\begin{array}{l}\text { And so ... Yeah, I mean we could keep plotting them. Or we could } \\
\text { Yeah, describe [inaudible] from here. }\end{array}$ & & & \\
\hline 74 & Matt: & $\begin{array}{l}\text { So then ... I guess there'd be sort of like a line of symmetry along [draws dotted } \\
\text { line along } y=x \text { ] this. }\end{array}$ & & \multirow{4}{*}{ Symmetry } & \multirow{6}{*}{ Sensemaking } \\
\hline 75 & Ian: & [Mm-hmm.] & & & \\
\hline 76 & Ben: & Yeah. & & & \\
\hline $\begin{array}{l}77 \\
78\end{array}$ & Matt: & And then also along here [draws a dotted line along $\mathrm{y}=-\mathrm{x}]$. & & & \\
\hline 79 & $\begin{array}{l}\text { lan: } \\
\text { Matt: }\end{array}$ & And you get some kind of symmetry where the vectors are going to converge on & & & \\
\hline & & to those lines. & & Extrapolate & \\
\hline
\end{tabular}

this activity, as well as columns indicating where we observe the moves of these different games. We transcribed coordinate pairs in the form $(\mathrm{x}, \mathrm{y})$ for readability, and included descriptions of the students' actions in square brackets within the transcript. We analyze these different layers to answer different research questions.

\section{A. The Pick, Plug, and Plot epistemic game}

We first look at some of the students' actions through the lens of the small grain-sized Pick, Plug, and Plot
$(P P P)$ epistemic game. The $P P P$ game is summarized in Table II. The epistemic form for $P P P$ is the twodimensional coordinate plane; the entry condition is the need to plot a vector on this plot (articulated by Ben (line 9 of Table I) when he suggests they "plot a couple points to get the feel of it"); and the ending condition is met when the point is plotted.

PPP has three moves; Pick, Plug, and Plot. In the Pick move, the students select a location at which to evaluate the vector field. In the Plug move, the students input the coordinates of this location into the formula and obtain an expression for the vector emanating from 
TABLE II. The Pick, Plug, and Plot epistemic game.

\begin{tabular}{ll}
\hline \hline Entry Cond. & Need to plot a single value \\
Ending Cond. & The plotting of the value \\
Form & The 2D coordinate plane \\
Moves & \\
Pick & Select a location at which to evaluate the formula \\
Plug & Obtain a value of the function from the formula \\
Plot & Interpret and plot the resulting value \\
\hline \hline
\end{tabular}

this point. In the Plot move, the students interpret this result geometrically and draw it on the coordinate plane.

We can use the fine-grained PPP to analyze smallscale details in the students' actions. The students move swiftly through this game, playing it to completion several times as they plot various points. However, there is one instance in which the students have difficulty: Ben incorrectly determines the length of a vector in the Plot move (line 54, Table I). Since Ben correctly found the function's output for this vector in the Plug move (line 49, Table I), we see that this is an error in the interpretation part of the Plot move. We surmise that Ben uses the function's output to determine the direction and magnitude of the vector, rather than interpreting the function's output as the $\mathrm{x}$ and $\mathrm{y}$ components of the vector.

\section{B. The Plotting epistemic game}

We might also describe the student's behavior at another layer by using the medium-grained Plotting epistemic game (Table II). In Plotting, the epistemic form is the coordinate plane; the entry condition is the prompt to plot the vector field; and the ending condition is when the students judge the plotting task complete (such as in line 72 of Table 1, when Ben says "I mean, we could keep plotting them. Or we could..."). It is possible that other moves, such as interpolation, could be employed in this game, though no such other moves were observed in this interview. We believe the moves of Plotting are applicable to any plotting activity, not just vector plotting.

Plotting's PPP move represents the 'chunking' of the moves of the small grain PPP game into a single move.

In the Extrapolate move, the students use the formula and patterns in the values already plotted to qualitatively predict the magnitudes and directions of vectors on the coordinate plane. In an early instance of this move, the students indicate that the vectors are larger further away

TABLE III. The Plotting epistemic game.

\begin{tabular}{ll}
\hline \hline Entry Cond. & Need to plot a function \\
Ending Cond. & A satisfactory plot representing the function \\
Form & The 2D coordinate plane \\
Moves & \\
PPP & As the PPP game, but a move \\
Extrapolate & Use plotted points to forecast trends \\
Symmetry & Use symmetry in the function or plot \\
\hline \hline
\end{tabular}

TABLE IV. The Answer Making with Sensemaking Game

\begin{tabular}{ll}
\hline \hline Entry Cond. & Asked a question or assigned a task \\
Ending Cond. & Successful communication of a sufficient solution \\
Form & Relevant equations and representations \\
Moves & \\
Conceptual & Consider the problem and relevant tools \\
Reasoning & Attempt to determine answer using relevant \\
Do Math & equations and mathematical procedures \\
Sensemaking & Create meaning based on math or reasoning \\
\hline \hline
\end{tabular}

from the origin. In lines 67-71 in Table I, the students are able to extrapolate from the existing points on the axes and in all four quadrants to describe the entire shape of the plot.

The Symmetry move manifests itself in two main ways. Initially, after the PPP move, the students use some basic symmetry to plot vectors situated across the origin from the vectors that they just plotted-we call this across-theorigin symmetry. Ben indicates several times that acrossthe-origin symmetry is not challenging for him, as he says (line 14, Table I) "and we know [how to plot these vectors]" without ever consulting the formula. Sometimes, such as in line 51 of Table I, the students even employ across-the-origin symmetry during the PPP move.

The students also use the Symmetry move when identifying the existence of axes of symmetry in the plot. After the group has plotted four points with PPP, four points with across-the-origin symmetry, and a few more via Extrapolation, they describe symmetry in the plot (lines 74-78 in Table I). This instance of Symmetry is purely descriptive and not used to plot more points, but they do employ it to describe the general pattern of the entire vector field.

\section{The Answer Making with Sensemaking epistemic game}

Chen et al. [4] describe their Answer Making Epistemic game as having "the ultimate goal [of producing] an answer." They contrast this game with sensemaking, the process of creating new knowledge. We believe the students in our interview play a variant of the $A n$ swer Making Epistemic Game, in which sensemaking (as a move) is a tool used to obtain an answer. Thus, we introduce Answer Making with Sensemaking (AMS) as an elaboration of Answer Making Epistemic Game.

The AMS game is summarized in Table IV. The student's entry condition is a prompt (they are asked to plot the vector field), and the students alternate between the Conceptual Reasoning move (picking points, extrapolating, using symmetry) and the Do Math move (using the formula, geometrically interpreting the results, plotting vectors). We also introduce the Sensemaking move, examples of which include the group's uses of patterns and symmetry. The Sensemaking move incorporates actions that go beyond simple recognition and reasoning found 
in Conceptual Reasoning and extends to the creation of new knowledge and understanding.

AMS also differs from the Answer Making Epistemic Game in the epistemic form and the ending condition. The ending condition for the Answer Making Epistemic Game would be the completed plot. However, the students' behaviors (such as in line 72 in Table 1) indicate their understanding of the ending condition of $A M S$ is that they must communicate their sensemaking and plotting abilities to the interviewer. Likewise, the epistemic form is not just the coordinate plane, but also the interactions with and feedback from the interviewer.

If we consider Ben's mistake in interpreting the length of a vector (line 54, Table I), we see that this mistake comes during the Do Math move in $A M S$. Since Do Math is procedural, we believe that the students view finding the exact length of the vector as routine, and thus that the mistake could have occurred simply because the students were not being careful. This belief is supported by Ben's statement that the vector's length is "not [the] square root of two." Since this length is actually the correct length, we believe that Ben's association of the "square root of two" with this vector indicates that he has the knowledge to get the correct answer.

\section{A MULTI-LAYERED ANALYSIS}

Different layers of epistemic games can be used productively on their own, but we can also consider multiple games simultaneously to gain an understanding of student behavior that is greater than the sum of these individual analyses. One example of this comes from using PPP and Plotting together. These games give us a flexible tool for analyzing the students' plotting procedure, including how they overcome mistakes, at multiple grain sizes. One could imagine a multitude of layered games that would allow a researcher to zoom in and out smoothly throughout the course of an analysis.

Considering $P P P$ and $A M S$ together, we see that the Pick move is part of Conceptual Reasoning, and that Plug and Plot moves are part of Do Math. Analyzing the Pick moves might help us better understand their ability to reason about vector fields than would analyzing the
Plug and Plot moves. In this interview, the students pick reasonable and productive points, so we would conclude they are experienced, if not expert, vector field plotters despite the math errors.

We can use the Plotting and $A M S$ games together to learn about the students' use of symmetry in this activity. In line 14 (Table I), Ben uses across-the-origin symmetry to plot a vector, and we classify this as Sensemaking, since Ben is creating knowledge (a new way to plot vectors on this plot). As the students plot more vectors, they continue to use across-the-origin symmetry, but do so without discussion (eg. line 51 of Table I). After the first instance of across-the-origin symmetry, the students incorporate this action in the Do Math move of $A M S$ (and thus into the PPP move of Plotting).

After plotting a number of points, Matt details the symmetry of the plot by drawing lines of symmetry. The interviewer did not solicit this information, nor did the group use this information to plot more points (which would indicate that they were still purposefully engaged in the Plotting game). However, this use of symmetry does give us (and the interviewer) some understanding of how Matt would continue plotting vectors, and thus we believe that Matt offers this explanation in an effort to satisfy his own ending conditions for the $A M S$ game.

\section{CONCLUSION}

We identified three new epistemic games: Pick, Plug and Plot; Plotting; and Answer Making with Sensemaking. We briefly demonstrated how each of these different layers of games could be used individually to answer different research questions. We then demonstrated that these different games can inform one another, and that considering multiple games simultaneously can yield results that are not obvious in the individual analyses.

Although a young framework, the epistemic games framework has promising implications for physics education research. With expansions, such as our consideration of concurrent games that differ by more than just grain size, researchers will be better able to explain student behavior, and instructors may have another tool with which to backwards design problems and activities.
[1] A. Collins and W. Ferguson, Educational Psychologist 28, 25 (1993).

[2] J. Tuminaro and E. F. Redish, Physical Review Special Topics-Physics Education Research 3, 020101 (2007).

[3] K. E. Black and M. C. Wittmann, in 2007 Physics Education Research Conference, Vol. 951 (AIP Publishing, 2007) pp. 53-56.

[4] Y. Chen, P. W. Irving, and E. C. Sayre, Physical Review Special Topics-Physics Education Research 9, 010108 (2013).

[5] M. B. Kustusch, D. Roundy, T. Dray, and C. A. Manogue,
Physical Review Special Topics-Physics Education Research 10, 010101 (2014).

[6] B. Modir, P. Irving, S. Wolf, and E. Sayre, in Physics Education Research Conference 2014, PER Conference (Minneapolis, MN, 2014) pp. 191-194.

[7] A. Pawlak, P. Irving, and M. Caballero, in Physics Education Research Conference 2015, PER Conference (College Park, MD, 2015) pp. 255-258.

[8] E. Redish, Teaching Physics with the Physics Suite (Wiley, 2003).

[9] S. H. Khandkar, University of Calgary 23 (2009). 